\title{
PREPARAÇÃO DE ELETRODOS OPTICAMENTE TRANSPARENTES
}

\author{
William da Silva Cardoso, Claudia Longo e Marco-Aurelio De Paoli*
}

Instituto de Química, Universidade Estadual de Campinas, CP 6154, 13084-971 Campinas - SP

Recebido em 5/1/04; aceito em 24/8/04; publicado na web em 17/2/05

\begin{abstract}
PREPARATION OF OPTICALLY TRANSPARENT ELECTRODES. A simple experiment for the preparation of transparent conducting glass electrodes by deposition of pure and fluorine doped $\mathrm{SnO}_{2}$ films is described. This procedure was tested in the undergraduate inorganic course at IQ-UNICAMP. The success in achieving a conducting layer was easily checked using the standard probes of a volt-ohm meter. The optical transmittance and thickness were studied by UV-vis spectrophotometry. To discuss the experimental results we place significant emphasis on molecular orbital and energy band model theories. The undergraduate students can also discuss the concepts related to the electronic properties of solids and to interesting new materials, such as transparent conducting films, which are the subject of significant current research and technological applications.
\end{abstract}

Keywords: FTO-glass; conductive glass; transparent electrode.

\section{INTRODUÇÃO}

Eletrodos opticamente transparentes são constituídos de um substrato de vidro, ou de poli (tereftalato de etileno), recoberto com um filme fino, condutor e transparente de óxido de estanho, $\mathrm{SnO}_{2}$, que pode ser dopado com flúor ou outros dopantes. Estes eletrodos encontram inúmeras aplicações tecnológicas e em laboratórios de pesquisa. Entre as aplicações tecnológicas, uma das mais comuns é o uso em portas de geladeiras de supermercados destinadas a expor um produto de forma visível ao consumidor (sorvetes, por exemplo). Como estas portas costumam embaçar com a umidade depois de abertas, usa-se um vidro condutor para aplicar uma pequena diferença de potencial para desembaçá-las quando são novamente fechadas. Também são usados em espelhos eletrocrômicos de veículos e em muitas outras aplicações. Nos laboratórios de pesquisa estes eletrodos são usados para depositar filmes finos de materiais eletrocrômicos e estudá-los por técnicas espectrofotométricas acopladas a técnicas eletroquímicas como, por exemplo, espectrocronoamperometria ${ }^{1}$. Estes eletrodos também são usados extensivamente para construir células fotoeletroquímicas de óxido de titânio sensibilizado por corantes ${ }^{2-4}$.

Os filmes finos de $\mathrm{SnO}_{2}$ tornaram-se materiais tecnologicamente importantes por possuírem uma alta transparência (comparados com outros óxidos) na região do visível, alta condutividade elétrica, boa estabilidade química e mecânica e uma forte interação com espécies adsorvidas ${ }^{5}$.

A escolha da rota de síntese de filmes finos de $\mathrm{SnO}_{2}$ é de vital importância nas propriedades do filme, principalmente se, através da rota, for possível otimizar parâmetros fundamentais, tais como transparência, condutividade, tamanho das partículas e composição do filme. Em geral, as propriedades dos filmes finos são fortemente influenciadas pela escolha do método de preparação. Dentre algumas das técnicas mais comuns de preparação de filmes finos de $\mathrm{SnO}_{2}$, incluem-se a deposição química de vapor, "spray” pirólise, deposição eletrostática de "spray", técnicas de "sputtering” e sol-gel ${ }^{6-10}$. Os materiais precursores do filme utilizados nestas técnicas incluem compostos de estanho, tais como $\mathrm{SnO}_{2}, \mathrm{SnCl}_{4}, \mathrm{SnCl}_{2}$ ou $\mathrm{SnR}$, onde $\mathrm{R}$ é um substituinte orgânico. Dentre estes, o mais utilizado é o $\mathrm{SnCl}_{4} .5 \mathrm{H}_{2} \mathrm{O}$ dissolvido em metanol ${ }^{11}$.

*e-mail: mdepaoli@iqm.unicamp.br
No presente trabalho apresentamos um experimento simples, possível de ser conduzido em um laboratório experimental de química contendo um mínimo de infra-estrutura, mas que propicie condições para os alunos se aprofundarem em conhecimentos teóricos básicos em química inorgânica. A estratégia do experimento compreende duas aulas práticas de $8 \mathrm{~h}$ cada, nas quais são abordadas diversas técnicas de laboratório, assim como os métodos de caracterização de filmes finos transparentes de $\mathrm{SnO}_{2}$.

A primeira aula consiste em sintetizar o $\mathrm{SnCl}_{4}$ através de uma reação em fase heterogênea gás/sólido, a partir da produção de gás cloro e posterior reação com estanho metálico. Na aula seguinte é feita a preparação do filme fino de $\mathrm{SnO}_{2}$ sobre substrato de vidro, usando o método de "spray" pirólise.

A opção pelo método de "spray” pirólise deve-se à sua simplicidade e baixo custo, tornando assim o experimento perfeitamente exequiível dentro de um laboratório de ensino. Os resultados apresentados foram obtidos pelos alunos do curso de Química Inorgânica Experimental II, oferecido pelo Instituto de Química da Unicamp, no primeiro semestre de 2003.

\section{PARTE EXPERIMENTAL}
Síntese do $\mathrm{SnCl}_{4}$
$\mathrm{O}$ sistema para síntese do $\mathrm{SnCl}_{4}$ deve ser montado de acordo com o esquema mostrado na Figura 1. No kitasato são colocados 50 g de $\mathrm{MnO}_{2}(0,71 \mathrm{~mol})$, no funil de adição são colocados $300 \mathrm{~mL}$ de $\mathrm{HCl}$ comercial, no frasco lavador coloca-se $100 \mathrm{~mL} \mathrm{de} \mathrm{H}_{2} \mathrm{SO}_{4}$ con- centrado e no reator coloca-se $8 \mathrm{~g}$ de $\mathrm{Sn}$ granulado $(0,07 \mathrm{~mol})$ (pre- viamente lavado com uma solução $0,1 \mathrm{~mol} \mathrm{~L}^{-1}$ de $\mathrm{HCl}$, água destila- da e álcool e seco em estufa). Antes de iniciar a reação verifica-se a vedação de todas as conexões e se todo sistema está isento de umida- de. Com auxílio do funil de adição inicia-se a lenta adição do $\mathrm{HCl}$ ao kitasato contendo $\mathrm{MnO}_{2}$, sob agitação constante e brandamente aque- cido (aproximadamente $70{ }^{\circ} \mathrm{C}$ ) para que a reação tenha início. O gás cloro formado é seco ao passar pelo frasco lavador e reagirá com o $\mathrm{Sn}$ granulado no reator até formar um composto líquido, o $\mathrm{SnCl}_{4}$. O reator deve ser mantido em banho de gelo para evitar superaqueci- mento e fusão do Sn metálico, que causaria entupimento do tubo de vidro por onde passa o gás cloro. Ao final de toda a reação transfere- 


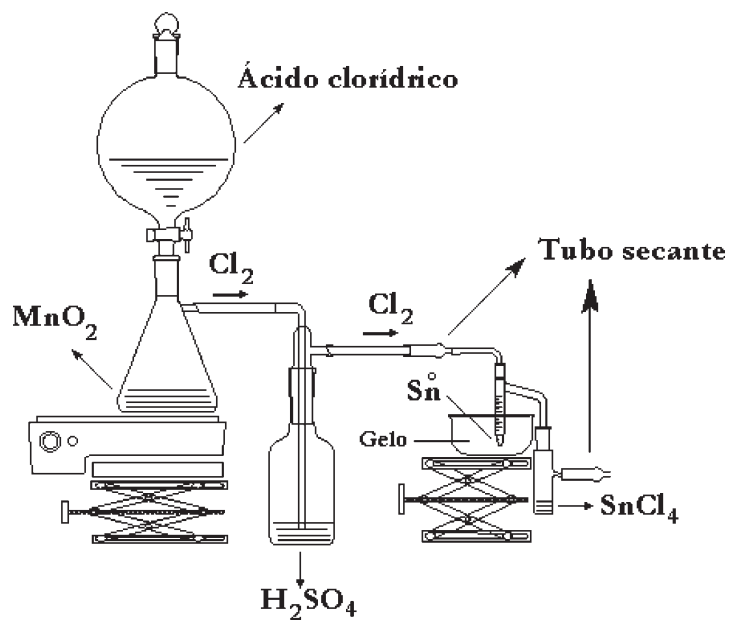

Figura 1. Esquema de montagem do sistema utilizado na síntese de $\mathrm{SnCl}_{4}$

se o $\mathrm{SnCl}_{4}$ obtido para um frasco coletor, previamente pesado. Pesase o $\mathrm{SnCl}_{4}$ formado e calcula-se o rendimento. Deve-se tomar o máximo cuidado ao manusear o $\mathrm{SnCl}_{4}$, pois ele reage instantaneamente com o ar. $\mathrm{O}$ cuidado com o meio ambiente deve ser observado e, portanto, devem ser reservados frascos para coleta dos resíduos das reações do processo. Os ácidos devem ser neutralizados antes de serem descartados.

\section{Preparação da solução precursora de $\mathrm{SnCl}_{4}$}

Prepara-se uma solução precursora de $\mathrm{SnCl}_{4}$, proporção 1:1 $\mathrm{SnCl}_{4} / \mathrm{CH}_{3} \mathrm{OH}(\mathrm{m} / \mathrm{v})$, pela pesagem de $15 \mathrm{~g}$ de $\mathrm{SnCl}_{4}$ e dissolução em $15 \mathrm{~mL}$ de metanol num Becker de $100 \mathrm{~mL}$. Para auxiliar na dissolução usa-se aquecimento suave com o auxílio de um banho-maria e, caso haja a formação de gel, deve-se filtrar a solução usando um funil de vidro sinterizado. $\mathrm{O}$ metanol deve ser adicinado sobre o $\mathrm{SnCl}_{4}$ lentamente, pois a reação que ocorre é vigorosa e exotérmica. A solução obtida deve ser dividida em duas partes, sendo uma reservada para a preparação do filme fino de $\mathrm{SnO}_{2}$ puro e a outra para preparação do filme de $\mathrm{SnO}_{2}$ dopado com flúor. Para a dopagem, adiciona-se $0,07 \mathrm{~g}$ de $\mathrm{NH}_{4} \mathrm{~F}$ à segunda parte da solução precursora de $\mathrm{SnCl}_{4}$. Alternativamente, essa dopagem pode ser feita usando 0,41 $\mathrm{g}$ de $\mathrm{SbCl}_{3}$ para dopagem com $\mathrm{Sb}$.

\section{Preparação do gerador de "spray"}

O gerador de "spray" pode ser feito de vidro ou pode-se usar um frasco análogo àqueles de aerosol para perfumes. A confecção do borrifador é relativamente simples e inclui um orifício de saída do "spray", medindo 2 a $3 \mathrm{~mm}$ de diâmetro, colocado na saída do tubo por onde o ar passará. O suprimento de ar de uma bomba comum de vácuo é suficiente para ser usado no borrifador.

\section{Preparação do filme transparente de $\mathrm{SnO}_{2}$}

O método consiste em aplicar através de um borrifador ("spray") uma solução de $\mathrm{SnCl}_{4}$ sobre o substrato de vidro pré-aquecido. A formação do óxido ocorre de acordo com a seguinte reação de hidrólise:

$\mathrm{SnCl}_{4}+2 \mathrm{H}_{2} \mathrm{O} \longrightarrow \mathrm{SnO}_{2}+4 \mathrm{HCl}$

A transcrição detalhada da preparação do filme fino de $\mathrm{SnO}_{2}$ é dada a seguir.
Manter próximo à capela uma estufa pré-aquecida a $100{ }^{\circ} \mathrm{C}$ e uma mufla a $600^{\circ} \mathrm{C}$. Usar um suporte para as lâminas de vidro feito em alumínio ou outro material resistente à temperatura de $600{ }^{\circ} \mathrm{C}$. Coloca-se uma lâmina de vidro no suporte e aquece-se por 5 min no forno a $100{ }^{\circ} \mathrm{C}$, em seguida o conjunto é transferido para o forno a $600{ }^{\circ} \mathrm{C}$ e aquecido por $15 \mathrm{~min}$. Com o auxílio de uma pinça metálica retira-se o conjunto (suporte/placa de vidro) do forno deixando-o na posição vertical e, imediatamente a seguir, borrifa-se a solução precursora por $3 \mathrm{~s}$ a uma distância de $10 \mathrm{~cm}$, recolocando o conjunto no forno a $600{ }^{\circ} \mathrm{C}$ durante $5 \mathrm{~min}$. Esta operação é repetida três vezes em uma lâmina e 6 vezes em outra lâmina, sendo que, no intervalo entre uma borrifada e outra, o suporte contendo a lâmina é levado de volta ao forno a $600{ }^{\circ} \mathrm{C}$ por $5 \mathrm{~min}$. Repete-se este procedimento usando a solução metanólica de $\mathrm{SnCl}_{4}$ contendo $\mathrm{NH}_{4} \mathrm{~F}$. Deve-se preparar uma lâmina de cada conjunto para poder fazer os testes descritos abaixo. No total serão 2 lâminas de vidro recobertas com $\mathrm{SnO}_{2}$ não dopado, obtidas com 3 e 6 borrifadas e 2 lâminas de vidro recobertas com $\mathrm{SnO}_{2}$ dopado com flúor, também obtidas com 3 e 6 borrifadas, em um total de 4 lâminas.

Os filmes de $\mathrm{SnO}_{2}$ sobre o substrato de vidro são caracterizados por medida da condutividade superficial e do espectro de absorbância na faixa de 300 a $1200 \mathrm{~nm}$. A medida na região de 700 a $1200 \mathrm{~nm}$ é necessária para o cálculo da espessura, usando as franjas de interferência. A medida do espectro do branco deve ser feita com uma lâmina de vidro limpa. Deve-se medir o espectro de uma amostra de cada conjunto de vidros obtidos e repetir a medida depois de fazer os tratamentos descritos abaixo para testar a resistência do material ao ataque por solvente ou ácidos. A medida do espectro de absorbância é feita posicionando-se as placas de vidro na vertical e perpendicularmente ao feixe de luz que passa pelo compartimento de amostra do espectrofotômetro. Em nosso laboratório usamos o espectrofotômetro UV-vis da Shimadzu modelo UV 1601 PC.

A resistividade superficial do filme é medida com um multímetro digital (por exemplo marca Minipa ET 2500), com fundo de escala adequado, com os eletrodos posicionados a $1 \mathrm{~cm}$ de distância um do outro. Para facilitar as medidas, coloca-se as placas sobre uma folha de papel com uma marca de escala de $1,0 \mathrm{~cm}$.

Para avaliar a resistência dos filmes de $\mathrm{SnO}_{2}$ ao ataque de ácidos e solventes, mergulham-se as placas, separadamente, em cada um dos seguintes solventes: água, álcool etílico, tolueno, e nos ácidos concentrados: clorídrico, sulfúrico e nítrico, a fim de se verificar se estes ácidos são nocivos à camada condutora do filme.

Todo o experimento dever ser realizado com os alunos portando os EPI adequados (avental e óculos de segurança são imprescindíveis) e em capela.

\section{RESULTADOS E DISCUSSÃO}

A preparação do precursor, $\mathrm{SnCl}_{4}$, é uma boa oportunidade para demonstrar para os alunos uma reação química em fase heterogênea (gás/sólido) sem o uso de solventes. Além disso, deve-se aproveitar a oportunidade para discutir o modelo de hibridização de orbitais atômicos em compostos inorgânicos.

$\mathrm{O} \mathrm{SnCl}_{4}$ é um composto com estrutura tetraédrica, onde as ligações sigma entre o estanho e os íons cloro podem ser explicadas pela interação entre os orbitais atômicos hibridizados $\mathbf{s p}^{3}$ do estanho e os orbitais p ocupados dos íons cloro. Muitas vezes os alunos de química têm a tendência a associar o modelo de hibridização $\mathbf{s p}^{3}$ somente ao carbono, esquecendo-se que este modelo também pode ser utilizado para explicar a ligação química em compostos inorgânicos de todos os elementos representativos.

As propriedades ópticas e elétricas das substâncias no estado sólido são tratadas baseando-se no modelo de bandas. Segundo esta 
teoria, a presença de infinitas espécies (átomos ou íons) nos retículos cristalinos, característicos de um sólido, faz com que os orbitais destas espécies interajam entre si, formando conjuntos de níveis de energia que recebem o nome de "banda". A banda de maior energia ocupada por elétrons é conhecida como "banda de valência", e a banda vazia de menor energia é denominada "banda de condução". A diferença de energia entre a banda de valência e a banda de condução é chamada de "band gap" (ou "banda proibida", ou "hiato"). A condutividade eletrônica de um sólido é função da possibilidade de ocorrer transferência de elétrons da banda de valência para a banda de condução, onde estes elétrons poderão movimentar-se livremente. Esta transferência requer energia suficiente para que os elétrons passem pelo "band gap". Conforme a magnitude deste, pode-se definir quatro tipos de sólidos: a) os condutores ou metais, onde não há "band gap" (a banda de valência e a banda de condução estão sobrepostas) e a condutividade elétrica aumenta com a diminuição da temperatura; b) os semicondutores, cuja banda de valência está totalmente ocupada e a banda de condução vazia, com um valor de "band gap" entre 0 e 3 eV e onde a condutividade elétrica aumenta com o aumento da temperatura; c) os semi-metais com banda de valência ocupada e banda de condução vazia e um valor de "band gap" próximo de zero (como a grafite por exemplo) e d) os isolantes, também com a banda de valência totalmente preenchida e banda de condução vazia, mas com um valor de "band gap" alto (maior que $5 \mathrm{eV}$ ), neste caso o composto se decompõe termicamente antes que seja atingida a situação energética de superação do "band gap". Nos semicondutores, pequenos valores de energia (térmica ou luminosa) possibilitam a transferência de elétrons para a banda de condução, fazendo com que estes materiais apresentem condutividade elétrica ${ }^{12}$.

A Tabela 1 apresenta os valores medidos da resistividade elétrica dos filmes finos de $\mathrm{SnO}_{2}$ não dopados e dos dopados com flúor, com três e seis aplicações, antes e após o tratamento com os diferentes solventes.

Os filmes de $\mathrm{SnO}_{2}$ mais espessos (seis aplicações do "spray”) apresentam menor resistividade quando comparados com o filme obtido a partir de três aplicações do "spray". A uniformidade e a espessura do filme de $\mathrm{SnO}_{2}$ é responsável por este resultado. $\mathrm{O} \mathrm{SnO}_{2}$ é classificado como um semicondutor do tipo-n, as bandas de valência e de condução são identificadas com os níveis $2 p$ do oxigênio (parcialmente preenchidos) e com os níveis $5 \mathrm{~s}$ do $\mathrm{Sn}$, respectivamente. O intervalo de energia da zona proibida é bem grande, $\mathrm{E}_{\mathrm{g}} \approx 3,5 \mathrm{eV}$, no entanto, esse material apresenta condutividade relativamente alta (especialmente quando está na forma de filme fino) ${ }^{13}$. Esse aumento na condutividade se deve, nesse caso, aos desvios na estequiometria do óxido formado. Os desvios na estequiometria aumentam a condutividade porque tanto as vacâncias de oxigênio, como a presença de átomos de $\mathrm{Sn}$ intersticiais, estão associadas a elétrons que podem se difundir pela estrutura do $\mathrm{SnO}_{2}$ formado ${ }^{14}$.
Analisando-se ainda a Tabela 1 pode-se observar que os valores de resistividade diminuem consideravelmente, para os filmes dopados com flúor. A troca de íons $\mathrm{O}^{2-}$ por $\mathrm{F}^{-}$altera a distribuição eletrônica das camadas de valência e de condução, aumentando a capacidade condutora do material. No caso dos dopantes, admitindo que uma fração x de sítios $\mathrm{Sn}^{4+}$ seja substituída por $\mathrm{F}^{-}$, x mols de elétrons poderiam ser doados à banda de condução. Para efeito ilustrativo, pode-se considerar que o óxido resultante teria a composição $\mathrm{Sn}_{(1-x)} \mathrm{F}_{x} e_{x} \mathrm{O}_{2}$. Existe ainda a possibilidade de que os dopantes se incorporem na estrutura do cristal intersticialmente ${ }^{15}$.

O flúor é um dos dopantes mais usados para aumentar a condutividade do $\mathrm{SnO}_{2}$. O principal motivo é que a introdução desta espécie não altera significantemente a estrutura cristalina do $\mathrm{SnO}_{2}$ devido à razoável similaridade entre os raios iônicos do ânion $\mathrm{F}^{-}$ $(1,33 \AA)$ e do $\mathrm{O}^{2-}(1.36 \AA)^{13}$.

Os valores de resistividade após a lavagem das lâminas contendo o filme fino de $\mathrm{SnO}_{2}$ em diferentes solventes não apresentaram diferenças significativas, quando comparados com os valores obtidos antes da lavagem. Isto indica que os filmes de $\mathrm{SnO}_{2}$ obtidos são quimicamente estáveis.

A partir dos resultados de medidas de espectrofotometria na região do UV-Vis-NIR (300 a $1100 \mathrm{~nm}$ ) é possível obter informações preciosas a respeito de propriedades interessantes do eletrodo opticamente transparente, tais como transparência, espessura do filme e energia de "gap".

A Figura 2 mostra os espectros de transmitância do filme de $\mathrm{SnO}_{2}$ dopado com flúor e dos filmes não dopados obtidos com três e seis aplicações do "spray”. A transmitância em $550 \mathrm{~nm}$ do filme não dopado e com três aplicações foi de $86 \%$ (Figura 2b) e, à medida que foi se aumentando a espessura do filme, a transmitância diminuiu para $57 \%$ (Figura 2d). Mede-se a transmitância em $550 \mathrm{~nm}$ porque este comprimento de onda corresponde à região do visível com maior sensibilidade para o olho humano. A transmissão óptica de um filme transparente de $\mathrm{SnO}_{2}$ obtido pela técnica de "spray" pirólise varia na faixa de 80 a $90 \%{ }^{1,13}$. A queda tão acentuada na transparência do filme devese ao aumento na espessura da camada do filme depositada sobre a lâmina e, principalmente, pelo modo como o mesmo foi preparado. Sabe-se que as propriedades físicas dos filmes dependem das condições de preparação, entre as quais a temperatura e as características do substrato, a concentração da solução precursora, a duração e o número de repetições de aplicação do "spray". Até mesmo a distância entre o "spray" e o substrato é um fator crítico, porque, se for muito curta, a reação não terá se completado e as espécies que não reagiram podem se incorporar ao filme e, se for muito grande, as gotículas podem vaporizar antes de chegar ao substrato, formando um pó que não adere ou sequer atinge o substrato ${ }^{16,17,18}$.

A partir do espectro de transmitância para o filme formado após seis aplicações do "spray" de $\mathrm{SnCl}_{4}$, Figura 2, nota-se que com seis

Tabela 1. Valores de resistividade dos filmes finos de $\mathrm{SnO}_{2} \operatorname{com} 3$ e 6 aplicações do "spray”. Antes e após tratamento com solventes. Medidas feitas com um multímetro com os eletrodos a $1 \mathrm{~cm}$ de distância

\begin{tabular}{lcccc}
\hline & $\begin{array}{c}\mathrm{A}^{*} \\
\text { aplicações/k } \Omega\end{array}$ & $\begin{array}{c}\mathrm{A} \\
\text { 6 aplicações/k } \Omega\end{array}$ & $\begin{array}{c}\mathrm{B}^{\#} \\
\text { aplicações/k } \Omega\end{array}$ & $\begin{array}{c}\mathrm{B} \\
\text { aplicações/k } \Omega\end{array}$ \\
\hline Sem lavagem & 6,4 & 0,5 & 1,5 & 0,1 \\
Água & 5,9 & 0,6 & 1,3 & 0,2 \\
Álcool etílico & 5,1 & 0,5 & 1,1 & 0,2 \\
Tolueno & 5,5 & 0,5 & 1,5 & 0,3 \\
$\mathrm{HCl}_{\text {(conc.) }}$ & 5,1 & 0,5 & 1,4 & 0,2 \\
$\mathrm{H}_{2} \mathrm{SO}_{4 \text { (conc.) }}$ & 4,2 & 0,5 & 1,3 & 0,2 \\
$\mathrm{HNO}_{3 \text { (conc.) }}$ & 4,9 & 0,5 & 1,3 & 0,2 \\
\hline
\end{tabular}

"Filme de $\mathrm{SnO}_{2}$ não dopado; "filme de $\mathrm{SnO}_{2}$ dopado com F-. 


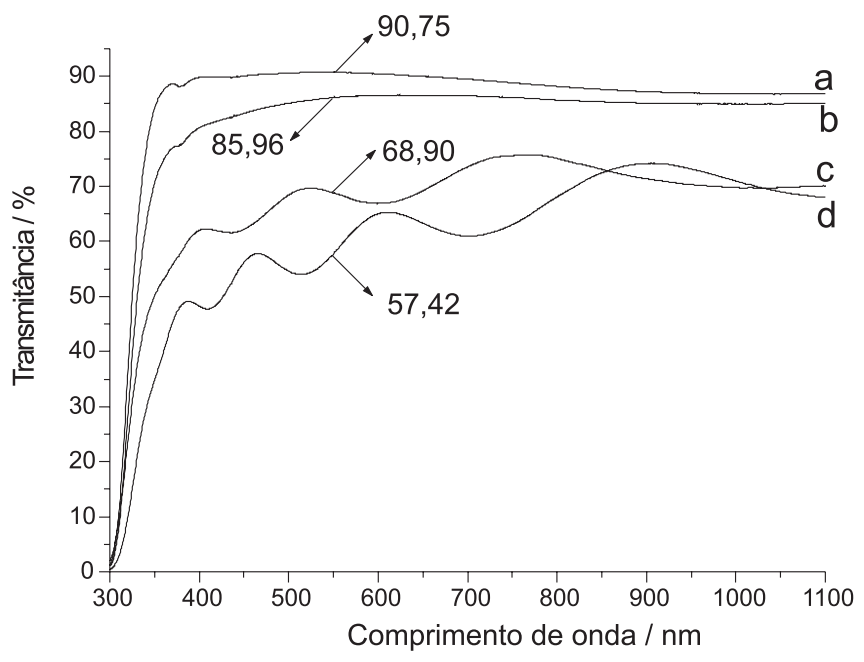

Figura 2. Espectros de transmitância dos filmes finos de $\mathrm{SnO}_{2}$ não dopado e dopado com flúor: (a) lâmina de vidro limpa sem filme, (b) filme fino com 3 aplicações não dopado, (c) filme fino com 3 aplicações dopado com flúor, (d) filme fino com 6 aplicações dopado com flúor

aplicações foi possível observar as franjas de interferências formadas (Figura 2c e 2d). A espessura do filme de $\mathrm{SnO}_{2}$ formado sobre a lâmina de vidro pode ser calculada pela Equação 1, onde t é espessura do filme em $(\mathrm{cm}) ; \mathrm{N}$ é o número de franjas encontradas entre $v_{1} \mathrm{e}$ $v_{2}$, que são as freqüências dadas em $\left(\mathrm{cm}^{-1}\right)^{19}$.

$2 \mathrm{t}=\mathrm{N} /\left(\mathrm{v}_{1}-\mathrm{v}_{2}\right)$

A energia do "gap" também é obtida do espectro de absorbância e é calculada pela Equação 2, onde $E=$ energia do "gap" em eV e $\lambda$ $=$ comprimento de onda em $\mathrm{nm}^{20}$.

$\mathrm{E}=1240 / \lambda$

A determinação do comprimento de onda é feita por extrapolação de uma reta, traçada tangente à curva de absorbância, até o eixo de comprimento de onda. Alguns espectrofotômetros interfaceados a computadores permitem a conversão da escala de comprimento de

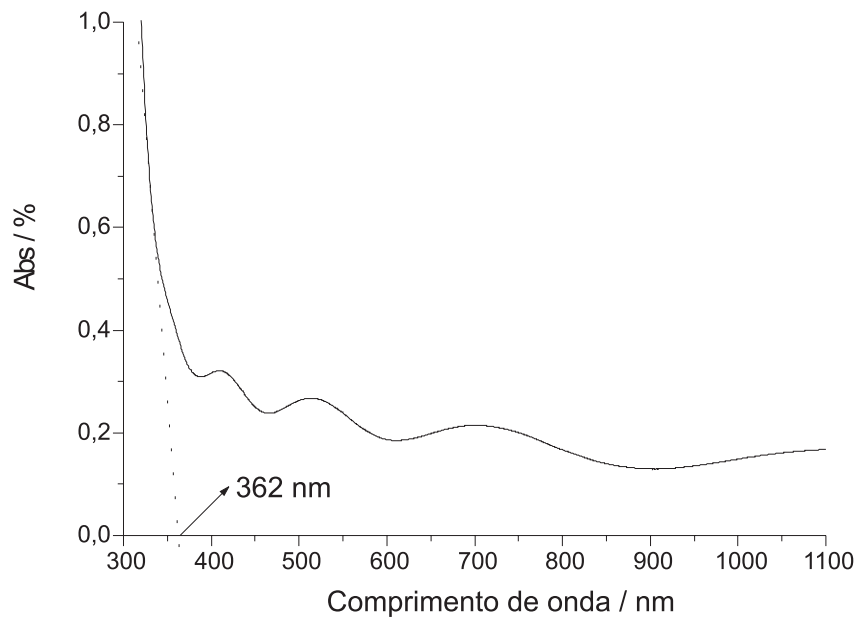

Figura 3. Espectro de absorbância de um filme de $\mathrm{SnO}_{2}$ não dopado após seis aplicações onda de nm para eV, dispensando o cálculo pela Equação 2. A Figura 3 é ilustrativa de um exemplo da obtenção do comprimento de onda a partir do espectro de absorbância de um filme de $\mathrm{SnO}_{2}$ não dopado após seis aplicações. O valor encontrado pelos alunos para energia de "gap" foi de $3,4 \mathrm{eV}$, valor muito próximo daquele encontrado na literatura que é $3,5 \mathrm{eV}^{18}$.

\section{CONCLUSÕES}

Na introdução deste trabalho deu-se ênfase à aplicação do filme fino opticamente transparente de $\mathrm{SnO}_{2}$ depositado em substrato de vidro, relacionando seu uso com o nosso cotidiano, tal como em portas de vidro de geladeiras de supermercados. Essa idéia de simplicidade é enfatizada para os alunos durante o transcorrer do experimento, vinculando a química envolvida neste contexto a exemplos do nosso dia-a-dia. A importância deste resgate do cotidiano dá-se principalmente quando podemos mostrar e discutir conceitos químicos vistos em sala de aula e que normalmente não são apreciados pelos alunos. Isto é importante especialmente para a química inorgânica, onde aplicações tecnológicas modernas são pouco observadas pelos estudantes.

Este experimento consta de duas etapas distintas onde se pode envolver a participação do aluno desde a preparação do precursor, no caso o $\mathrm{SnCl}_{4}$, até a obtenção do produto final e se discutir e aplicar modelos de ligação química, como hibridização de orbitais atômicos em compostos tetraédricos de elementos representativos, modelo de bandas, estrutura cristalina de sólidos e criação de defeitos a partir da dopagem. Além disso, mostra o uso de técnicas simples para obtenção de novos materiais. A discussão do modelo de ligações químicas aplicado ao composto de estanho, $\mathrm{SnCl}_{4}$, é particularmente importante para vincular o modelo de orbitais hibridizados a compostos inorgânicos.

Vale ressaltar a importância de se discutir na prática o uso da técnica de espectrofotometria UV-vis, interpretar um espectro de absorção do filme na região do ultravioleta-visível e, a partir dele, tirar informações imprescindíveis para a caracterização do filme. Por outro lado, a impossibilidade do uso da espectroscopia UV-vis não compromete a compreensão e interpretação dos resultados obtidos; de modo geral, eles só ficariam incompletos. Por exemplo, diferenciar um filme dopado de um não dopado pode ser feito simplesmente pela medida da resistência ôhmica superficial do filme.

Convém registrar que apresentar e discutir os resultados deste experimento requer do aluno de graduação um mínimo de conhecimento básico em química inorgânica, necessário para o entendimento dos objetivos do trabalho. Portanto, é preciso levar em conta os aspectos relacionados aos pré-requisitos para sua realização, sendo importante que o aluno já tenha tido disciplinas de química inorgânica teórica, além de ter concluído a disciplina de química analítica quantitativa.

Finalizando, o laboratório de ensino é também o local perfeito para despertar a consciência do aluno de química para os cuidados com o meio ambiente, mostrando a importância e a necessidade de se recolher, tratar e descartar adequadamente os resíduos gerados em cada etapa do trabalho. Esses aspectos devem ser levados em consideração em todas as etapas do procedimento experimental.

\section{AGRADECIMENTOS}

W. S. Cardoso agradece à FUNCAMP pela concessão do estágio de capacitação docente junto ao IQ-UNICAMP e ao CNPq pela bolsa de doutoramento recebida. M.A De Paoli agradece ao CNPq a bolsa de produtividade acadêmica. 


\section{REFERÊNCIAS}

1. Girotto, E. M.; Gazotti, W. A.; Martini, M.; Micaroni, L.; das Neves, S.; De Paoli, M.-A. Em Electroquímica y Electrocatalisis; Alonso-Vante, N., ed.; Editorial Ciudad Educativa: Cordoba, 2003, vol. IIa, cap. 13, p. 1966

2. Longo, C.; De Paoli, M.-A.; J. Braz. Chem. Soc. 2003, 14, 889.

3. Härtinger, S.; Machado, D. A.; De Paoli, M.-A.; Quim. Nova 1997, 21, 343.

4. Gazotti, W. A.; Nogueira, A. F.; Girotto, E. M.; Micaroni, L.; Martini, M.; Das Neves, S.; De Paoli; M.-A. Em Handbook of Advanced Electronic and Photonic Materials; Nalwa, H. S., ed.; Academic Press: San Diego, 2000, vol. 10 , p. $54-98$

5. Greenwood, N. N.; Earnshaw, A.; Chemistry of the Elements, Pergamon Press: New York, 1984, p. 427.

6. Tamura, S.; Ishida, T.; Magara, H.; Mihara, T.; Mochizuki, S.; Tatsuta, T.; Appl. Surf. Sci. 2001, 169-170, 425.

7. Orel, B.; Lavrencic-Stangar, U.; Crnjak-Orel, Z.; Bukovec, P.; Kosec, M.; J. Non-Cryst. Solids 1994, 167, 272.

8. Huang, H.; Kelder, E. M.; Chen, L.; Schoonman, J.; Solid State Ionics 1999, $120,205$.
9. Kobayashi, H.; Uebou, Y.; Ishida, T.; Tamura, S.; Mochizuki, S.; Mihara, T.; Tabuchi, M.; Kageyama, H.; Yamamoto, Y.; J. Power Sources 2001, 97. 98, 229

10. Gubbins, M. A.; Casey, V.; Newcomb, S. B.; Thin Solid Films 2002, 405, 270.

11. Lee, C. H.; Liu, S. H.; Thin Solid Films 1992, 219, 170.

12. Shriver, D. F.; Atkins, P.W.; Langfortt, C.H.; Inorganic Chemistry, Oxford University Press: Oxford, 1994.

13. Nanthakumar, A.; Armstrong, N. R. Em Semiconductor Electrodes; Finklea, H. O., ed.; Elsevier: Amsterdan, 1988, cap. 4.

14. Smart, L.; Moore, E.; Solid State Chemistry - An Introduction, Chapman \& Hall: Londres, 1995, caps. 2 e 3.

15. Vicent, C. A.; J. Electrochem. Soc.1972, 119, 515.

16. Tanaka, J.; Suib, S. L.; J. Chem. Ed. 1984, 61, 263.

17. Agashe, C.; Marathe, B. R.; Takwale, M. G.; Bhide, V. G.; Thin Solid Films 1988, 164, 263.

18. Longo, C.; Tese de Doutorado, Universidade de São Paulo, Brasil, 1998.

19. Colthup, N. B.; Wiberley, S. E.; Daly, L. H.; Introduction to Infrared and Raman Spectroscopy, Academic Press: London, 1964, p. 62.

20. Ibanez, J. G.; Solorza, O.; Gomes-del-Campo, E.; J. Chem. Ed. 1991, 68, 872 . 